\title{
¿EL ATRIBUTO O EL COMPLEMENTO PREDICATIVO?
}

\begin{abstract}
Pawlik Janusz, ¿El atributo o el complemento predicativo? [The complement or the predicative complement?]. Studia Romanica Posnaniensia, Adam Mickiewicz University Press, Poznań, vol. XXXI: 2004, pp. 423-431. ISBN 83-232-1353-4, ISSN 0137-2475.

The purpose of this paper is to examine the difference betwcen the complement and the so-called predicative complement in Spanish. Traditionally grammarians distinguish in this language the predicative complement as a different syntactic function due to the existence of some specific, typically Spanish, constructions in which the subject and the verb are complemented at the same time. We hereby intend to find out if this double modification is the real feature of the predicative complement.
\end{abstract}

En la presente comunicación pretendemos analizar dos funciones oracionales, atributo y complemento predicativo (CP), que a pesar de formar parte del predicado oracional van estrechamente unidas a elementos nominales de la oración. Tomamos como punto de referencia el capítulo dedicado al complemento predicativo en la Gramática descriptiva de la lengua española (1999) cuyos autores Violeta Demonte y Pascual José Masullo tratan dicha problemática de manera más exhaustiva y detallada que cualquier otra monografía publicada hasta el momento.

Los conceptos de atributo y predicativo que nos interesan aquí tienen su historia. Este último apareció en la gramática de la RAE (1973), pero con una acepción más amplia, abarcando también en su contenido la categoría que hoy conocemos como atributo. En el apartado titulado Oraciones de predicado nominal (RAE 1973: 364) leemos:

Por ejemplo, la oración mi padre está enfermo se analiza así: mi padre (sujeto), está enfermo (predicado), que a su vez se analiza así: está (verbo) y enfermo (complemento predicativo). En la oración estos jovenes son estudiantes distinguimos: estos jóvenes (sujeto) son estudiantes (predicado); y en el predicado: son (verbo) y estudiantes (complemento predicativo).

Esta postura la encontramos todavía en Manuel Seco (1991: 120) que la explica del modo siguiente: 
El nombre que suelen darle los gramáticos es cl de 'atributo'. Pero, como esta denominación la emplean otros para designar el adjetivo unido al nombre (p.ej. ojos claros), es preferible, para evitar toda confusión, el término 'predicativo', utilizado por varios autores modernos.

La base de distinción entre atributo y $\mathrm{CP}$ reside, según la mayoría de los autores, en la diferente relación que se mantiene entre estos modificadores y el verbo. Reconocemos el atributo en las oraciones del predicado nominal que contienen un verbo copulativo (ser, estar, parecer), p. ej.:

Mi padre está enfermo,

Estos jovenes son estudiantes,

Nuestro coche parece aquél,

en las que el atributo 'enfermo', 'estudiantes' y 'aquél' complementa el sintagma nominal en la función de sujeto. Otra cosa es cuando la oración cuenta con un predicado verbal, y junto al verbo predicativo aparece un modificador del sujeto, según se observa en:

Sus padres vivian felices.

Los huéspedes llegaron cansados.

Los grámaticos afirman simplemente que el atributo modifica sólo al sujeto mientras que el CP tanto al sujeto como al verbo; "Se llama así al CP al adjetivo que funciona a la vez como atributo del sujeto (concuerda con él) y como complemento del verbo" (Lázaro 1993: 68). Esta opinión la comparten otros lingüístas como C. Hernández Alonso (1986), M. Porroche Ballesteros (1990) y muchos otros. La misma interpretación como el criterio selectivo básico se encuentra en la ya citada GDLE (1999: 2463-2464) donde V. Demonte y P. J. Masullo aplican una definición parecida, caracterizando al CP:

Denominamos 'complementos predicativos' a aquellos constituyentes que modifican simultáneamente al predicado verbal y a un sintagma nominal de la misma oración (típicamente, al sujeto y al objeto directo sintáctico), con cuyo núcleo concuerdan en género y número. (...) Son complementos predicativos aquellos constituyentes que modifican a la vez al predicado verbal y a un argumento de la oración, siendo el caso que determinados verbos principales seleccionan el par de elementos formado por el predicativo y un sintarma nominal.

Nos gustaría expresar algunas reservas acerca del criterio formulado más ariba. Surgen dudas en cuanto a la posibilidad de modificar el verbo predicativo por un CP entendido así en algunos casos. Pónganse tres ejemplos sacados del estudio en cuestión:

La niña llegó cansada a casa.

Las aguas bajan turbias.

Irene sonrió contenta.

Los adjetivos 'cansada', 'turbias' y 'contenta' se refieren a los sintagmas nominales en función de sujeto, 'la niña', 'las aguas', 'Irene', y acompañan a los verbos 
predicativos 'llegar', 'bajar' y 'sonreír', es decir, con pleno significado léxico. Sin embargo, en realidad, ninguno de los calificativos modifica ni complementa al verbo ni al predicado verbal. El supuesto $\mathrm{CP}$ se refiere sólo a las formas nominales, o sea, en este caso al sujeto. Es como si dijésemos:

La médica, cansada, llegó a casa.

Las aguas, turbias, bajan.

Irene, contenta, sonrio.

Los autores comentan al respecto que: "En efecto, el predicativo 'contenta' indica un estado de 'Irene' (predica una propiedad transitoria de ella) y expresa a su vez que ese estado se manifiesta asociado al acto de sonreír" (ibidem, 2464). Esta asociación nos parece lejana y sería muy forzado hablar entonces de una modificación del predicado verbal.

A veces existe una conexión que relaciona el adjetivo con el verbo antepuesto, y por tanto los adjetivos incisos, como en las frases precedentes, no expresan exactamente el mismo sentido. Es posible que se trate entonces de una relación de tipo cronológico-causal: la niña llegó cansada a casa (quizás a resultas de un largo viaje), mientras que en: la niña, cansada, llegó a casa, no se sugiere necesariamente que cl cansancio de la niña estuvicra relacionado con el hecho de llegar. Pero esto no significa que la función que descmpeña el adjetivo en ambas posiciones sea diferente. Es evidente que el orden de palabras influye ocasionalmente en la reinterpretación semántica de la oración entera, pero la función que desempeña una palabra dada, a pesar de su posición, sigue siendo la misma. Veamos cómo el lugar del complemento circunstancial puede incidir sobre el sentido de las siguientes oraciones:

En Madrid, todos son simpáticos.

Todos son simpáticos en Madrid.

No cabe duda que los adjetivos no cumplen aquí función de complemento circunstancial de modo respecto al verbo, como sucede en otros casos, p. ej.: El chico contestó nervioso (= nerviosamente); Irene sonrió feliz (= felizmente). Hay que reconocer que esta característica atributiva de algunos CPs la advierten también los autores de la monografía que señalan lo siguiente:

Ese significado de manera, incidentalmente, era considerado por una buena parte de los estudios tradicionales como una característica general de todos los predicativos (1999: 2478). (...) Hemos indicado que la lectura de manera sólo se da con los predicativos orientados al sujeto y entre ellos, característicamente, con los que concurren en oraciones con verbos transitivos e intransitivos de actividad en cuya cstructura léxica están presentes componentes que expresan duración de un proceso, sea por el significado interno del verbo o porque la acción se realiza sobre algo. (...) En suma, la acepción atributiva básica - común a todos los predicativos y definitoria de ellos - se sigue de la naturaleza de 'eventivos o transitorios' de los predicados que constituyen la relación de predicación, mientras que la acepción adverbial superpuesta a ella depende de la composición léxica de los significados de adjetivos y verbos (ibidem, 2482). 
Es verdad que la lectura puramente atributiva o atributivo-adverbial de los llamados predicativos depende de factores semánticos complejos. Sin embargo, según los autores de la obra, también en el primer caso el predicativo viene a modificar el predicado verbal de la oración. En el caso contrario, es obvio que no calificarían a tales adjetivos como CPs por no cumplir el criterio selectivo de 'modificación'. Los autores niegan, además, la equivalencia de los CPs orientados al sujeto con los adjetivos incisos en la oración que para nosotros es un hecho (ibidem, 2483). Consideremos dos ejemplos oportunos:

Nos paramos jadeantes y sudorosos sobre la hierba fresca.

Jadeantes y sudorosos, nos paramos sobre la hierba fresca.

Dionisio recorrió cinco kilómetros sonriente.

Dionisio, sonriente, recorrí cinco kilómetros.

Repetimos que, aunque en el primer caso los calificativos 'jadeantes y sudorosos' y 'sonriente' siguen al verbo, se refieren y forman parte del predicado nominal y no verbal (nosotros estábamos jadeantes y sudorosos; Dionisio era sonriente). Igual de confusas parecen ser las relaciones internas dentro de las construcciones con los verbos pseudo-copulativos que en opinión de los autores del estudio pasan por estructuras predicativas. Así, p. ej., aparecen predicativos en oraciones que otros gramáticos prefieren considerar atributivas:

María se puso/cayó enferma.

Luis se volvió/quedó inútil después del accidente.

Evidentemente, la relación entre el verbo y el adjetivo aparece tan estrecha como en el caso de los predicados nominales y no puede compararse con las que se manifiestan ocasionalmente en los casos expuestos antes. Sin los calificativos, las oraciones citadas se hacen incompletas, igual que sucede con las oraciones copulativas: María se puso; María es/está.

Demonte y Masullo distinguen con razón los CPs orientados no sólo al sujeto, sino también al objeto. Estos últimos adquieren, igual que los primeros, la forma nominal, es decir, pueden ser sintagmas adjetivos, sustantivos, pronominales, preposicionales, etc. Los CPs en forma de adjetivo van en cursiva en los ejemplos siguientes:

Considero a tu hermana inteligente.

Encuentro tu propuesta may original.

El juez te declara culpable.

Los CPs 'inteligente', 'original' y 'culpable' califican a los objetos directos 'a tu hermana', 'tu propuesta' y 'te', pero no existe ninguna conexión que los una con los verbos 'considerar', 'encontrar' y 'declarar'. No entendemos cómo un adjetivo podría complementar al verbo. Los predicativos vinculados al objeto directo van muy frecuentemente en forma sustantiva, como en: 
Nombraron a Julio (como) delegado de curso.

Considero a Beatriz (como) la mejor alcaldesa.

Eligieron a Bush (como) presidente.

Los sintagmas 'delegado de curso', 'la mejor alcaldesa' y 'presidente' ciertamente no expresan ninguna circunstancia modal de realizarse la acción de los verbos mencionados ('nombrar, considerar, elegir').

Por otra parte, es verdad que se percibe cierta relación entre los verbos enumerados y los sintagmas adyacentes: nombrar delegado de curso, considerar la mejor alcaldesa y elegir presidente, que parecen bastante unidos dentro del mismo predicado verbal. Vemos, naturalmente, que el supuesto CP no modifica al predicado verbal del mismo modo que lo hacen algunos CPs orientados al sujeto. Su asociación con el verbo es además aparente y deriva de la elipsis que se produce en este tipo de construcciones. En realidad, frases simples como las transcritas arriba son meras abreviaciones de las compuestas donde las relaciones de dependencia se muestran con mayor nítidez:

Considero a tu hermana inteligente $=$ Considero que $u$ hermana es inteligente.

El juez te declara culpable $=\mathrm{El}$ juez declara que eres culpable.

Eligieron a Bush presidente $=$ Eligieron a Bush y Bush llegó a ser presidente .

Los elementos calificativos que se manifiestan en estas oraciones se refieren a los objetos que conjuntamente constituyen un tipo de enunciado no oracional (predicación secundaria). Sólo el todo forma parte del predicado verbal cuyo núcleo son verbos 'considerar', declarar', 'elegir'. En este sentido podemos decir que las expresiones 'a tu hermana inteligente', 'te culpable', 'a Bush presidente' modifican al predicado verbal, y no los predicativos aislados. En el caso contrario, no sonarían truncadas las frases: Eligieron como presidente, Nombraron como delegado de curso que necesitan un objeto directo (cf. Alarcos 1999: 307).

Es de notar que los verbos de estas oraciones se acercan semánticamente a los copulativos, ya que su significado léxico sirve de nexo para la predicación nominal. Son, en definitiva, palabras que contienen el sentido elemental del verbo copulativo parecer, aunque con ciertos matices adicionales. Consideremos la equivalencia de las siguientes construcciones:

Considero a Beatriz la mejor alcaldesa = Beatriz parece la mejor alcaldesa .

Te encuentro muy deprimido $=$ Pareces muy deprimido.

Por otro lado, la transformación a la pasiva de las oraciones con los verbos designativos 'nombrar', 'elegir', etc. descubre la afinidad del predicado compuesto pasivo 'ser nombrado' a la perífrasis semi-copulativa llegar a ser, lo cual se deja ver claramente abajo:

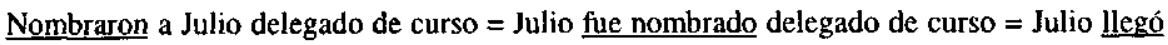
a ser delegado de curso por el nombramiento.

En consecuencia, es una razón más para que los adyacentes del objeto en los ejemplos enumerados sean tratados más bien como complementos atributivos, y no CPs. 
Volvamos ahora a la relación que guarda el CP con el verbo predicativo. La tesis según la cual el primero ejerce la función complementaria o modificadora respecto al segundo resulta de cierto tipo de frases, muy comunes en español, en las que esto efectivamente se lleva a cabo. Veámoslo:

El enfermero cruzo silencioso el pasillo.

Luisa respondió a todo nerviosa.

El guarda nos vigilaba atento en el parque.

Los elementos 'nerviosa', 'silencioso' y 'atento' se asemejan en cuanto al signifjcado gramatical a los complementos circunstanciales de modo 'silenciosamente, 'nerviosamente' y 'atentamente'. Conservando morfológicamente la forma adjetival, hacen las veces de los adverbios. Pero, como apuntan V. Demonte y P. J. Masullo, no conviene confundirlos con los adverbios adjetivales del tipo de los que aparecen en Habla fuerte y Juega limpio, que son sólo modificadores del verbo (1999: 2471). Estructuras de esta índole donde el adjetivo desempeña una doble función sintáctica constituyen una de las características especiales del castellano. Son también frecuentes en italiano y portugués. Con todo, las oraciones que imitan este modelo morfosintáctico no suenan correctas en otros idiomas, como lo vemos en la traducción del primer ejemplo al inglés y al polaco:

*The nurse crossed the corridor silent.

*Pielęgniarz przeszedl cichy korytarzem.

*Luise answered it all nervous.

*Luiza odpowiedziała na wszystko nerwowa.

Las normas sintácticas de ambas lenguas requieren que se usen en este caso las formas adverbiales referidas exclusivamente al predicado verbal, es decir:

The nurse crossed the corridor silently.

Pielęgniarz przeszedł cicho korytarzem.

Luise answered it all nervously.

Luiza odpowiedziała na wszystko nerwowo.

Esto no quiere decir que las formas adjetivales estén ausentes en dichas lenguas. Examinemos la semejanza formal de las tres oraciones siguientes:

El médico llegó cansado.

The doctor arrived tired.

Lekarz przyjechal zmęczony.

Lo que pasa es que, como ya hemos indicado, el adjetivo adyacente marcado en cursiva no puede definirse como un predicativo por falta de conexión sintáctica con el verbo. A nuestro juicio, debería tratarse más bien como atributo o complemento atributivo a pesar de que los verbos que intervienen no son típicamente copulativos. 
Al contrario del español, construcciones parecidas en otros idiomas se llaman atributivas. Tal enfoque es característico de la gramática francesa, que no distingue una función que sea comparable con el $\mathrm{CP}$ castellano. Según la Grammaire du Français Contemporain (1990: 81 ), los verbos que rigen el atributo pueden ser no sólo verbos copulativos (être, paraitre), sino tambien semipredicativos o predicativos, como p. ej. devenir, rester, demeurer, vivre, mourir, tomber, se trouver, se faire, se nommer, etc. El atributo puede orientarse tanto al sujeto (attribut du sujet) como al objeto (attribut de l'objet). He aquí algunos ejemplos oportunos recogidos por G. Mauger (1968: 57-58):

La classe devient attentive.

Il s'endort soldat, il se réveille roi.

Il est sorti, furieux.

On le nomme directeur.

Je te crois honnête.

Nous le tenons pour heureux.

Similar decisión terminológica toman los españoles Gutiérrez Ordóñez (1986) y Alarcos Llorach (1994) al referirse a estas construcciones como 'atributo' del sujeto, del objeto o de otros complementos. E. Alarcos en su Gramática de la Lengua Española apunta que incluso en las oraciones con la doble función modificadora del adjetivo calificativo no conviene hablar de un predicativo, sino de un adyacente atributivo, según se advierte en los ejemplos transcritos abajo (1994: 305):

El abogado vivía tranquilo.

Los árboles crecían lozanos.

Las olas rugen furiosas.

El término de 'adyacente atributivo' (dopełnienie orzecznikowe) es conocido también en la lengua polaca, pero como un elemento que completa únicamente el significado del objeto directo. Lo encontramos en las frases ya citadas en francés: Je le crois honnête; On l'a nommé directeur; On l'a choisi comme roi, que se traducen al polaco como:

Uważam go za uczciwego.

Mianowano go dyrektorem (na dyrektora).

Wybrano go królem (na króla).

El concepto de $\mathrm{CP}$ es un término descriptivo, tomado de una larga tradición, y que resulta de la existencia en la lengua española de estructuras sintácticas muy específicas, ajenas o poco usadas en otros idiomas (inglés, polaco, francés). Nos referimos a las oraciones predicativas antes citadas con el complemento adjetivo concordado con el sujeto y en función parecida al complemento circunstancial:

Los niños duermen tranquilos.

Ese señor colecciona afanoso braseros antiguos. 
Ha de quedar claro que en esta comunicación no hemos buscado eliminar dicho término de la lista de las funciones oracionales en español, sino abogar por una más estricta observación del criterio selectivo ya formulado que deberían cumplir necesariamente las unidades que han venido en llamarse CPs. Secundariamente, hemos reflexionado sobre el estatus gramatical de los elementos que comúnmente pasan por predicativos sin cumplir debidamente el criterio básico. En nuestra opinión, éstos se asemejan más a los complementos atributivos que predicativos. Por otra parte, existe la posibilidad de plantear otro criterio o criterios de selección que permitan incluir entre los predicativos las unidades que actualmente no merecen plenamente este apelativo.

Nos parece inopotruno seguir aplicando el viejo criterio de 'modificación' para determinar la categoría examinada. Sería deseable destacar una particularidad formal y más universal del $\mathrm{CP}$, como la de aparecer junto a los verbos predicativos. Además, tal complemento, en forma de adjetivo o sustantivo, habría de depender de un sintagma nominal de la misma oración (típicamente del sujeto o del objeto directo sintácticos). La modificación del sintagma verbal no se exigináa entonces como condición sine qua non.

En los estudios modernos de varias lenguas, incluido el polaco, aparece otro concepto de CP (określenie predykatywne) referido a los elementos nominales de construcciones como: Wracam królem 'Vuelvo como rey'; Przyszedl zmęczony 'Llegó cansado'. Se los concibe simplemente como exponentes de la predicación secundaria. Los complementos 'królem' i 'zmęczony' aparecen como enunciados no oracionales, formas elípticas de las frases compuestas: Wracam+jestem królem; Przyszedl + byl zmęczony (cf. Encyklopedia języka polskiego, 1991: 265-266). Merece la pena notar que esta conceptualización del CP es mencionada ya por C. Hernández Alonso (1986: 492) y la encontramos en las líneas siguientes:

Refuerza más esta conexión la existencia de unidades bivalentes en la lengua con función de modificador, a la vez, de sintagma nominal y sintagma verbal: es el llamado complemento predicativo, de forma y concordancia adjetiva, pero con esa doble función (ejemplos: Mis amigos viven contentos aquí). Se percibirá que, semánticamente, es el resultado de un cruce de las dos frases siguientes: mis amigos viven aquí + mis amigos están contentos.

\section{BIBLIOGRAFÍA}

A larcos Llorach, E. (1994), Gramática de la lengua española. Madrid: Espasa Calpe.

Encyklopedia jezyka polskiego, (1991), Stanisław Urbańczyk (ed.). Wrocław-Warszawa-Kraków: Ossolineum.

Demonte V., Masullo P. J. (1999), La predicación: los complementos predicativos, en Demonte V., Bosque I. (eds), Gramática Descriptiva de la lengua española, RAE Colección Nebrija y Bello. Madrid: Espasa Calpe, 2461-2523.

Grammaire du français contemporain (1990), J-C. Chevalier y otros. Paris: Larousse. 
Gutiérrez Ordoñez, S. (1986), Variaciones sobre la atribución. León: Ediciones de la Universidad de León.

Hernández Alonso, C. (1986), Gramática funcional del español. Madrid: Editorial Gredos.

Mauger, G. (1968), Grammaire pratique du français d'aujourd'hui. Paris: Librairie Hachette.

Lázaro, F. (1993), Lengua española COU. Madrid: Anaya.

Porroche Ballesteros (1990), Aspectos de la atribución en español. Zaragoza: Libros Pórtico.

RAE (1973), Esbozo de una nueva gramática de la lengua española. Madrid: Espasa Calpe.

Seco, M. (1991), Gramática esencial del español. Madrid: Espasa Calpe. 